\section{Metastasiertes Pankreaskarzinom: Deutliche Überlebensverlängerung durch Erlotinib bei Auftreten von Hautausschlag}

Die auf dem Symposium für Gastrointestinale Karzinome (ASCO GI) vorgestellte internationale Phase-III-Studie AViTA hat gezeigt: Patienten mit metastasiertem Pankreaskarzinom, die unter der Therapie mit Gemcitabin plus Erlotinib einen Hautausschlag («rash») entwickeln, leben signifikant länger als Patienten, die keinen Ausschlag entwickeln. Die Überlebensverlängerung konnte auch für Patienten mit nur leichtem Hautausschlag (Grad 1) nachgewiesen werden. Damit unterstreicht diese Studie die bisherige Empfehlung, Patienten mit metastasiertem Pankreaskarzinom mit der Kombination Gemcitabin plus Erlotinib anzubehandeln und diese Behandlung fortzusetzen, wenn nach 4-8 Wochen ein Hautausschlag auftritt.
Bisheriger Standard bei fortgeschrittener Erkrankung war eine Gemcitabin-Monotherapie. Die Kombination von Gemcitabin mit einem 2. Therapeutikum bot wenig zusätzlichen Nutzen. Vor 2 Jahren wurde jedoch eine Phase-III-Studie publiziert, die zeigen konnte, dass die Kombination von Gemcitabin mit dem oralen Tyrosinkinase-Inhibitor Erlotinib (Tarceva ${ }^{\circledR}$ ) das Überleben von Patienten mit fortgeschrittenem Pankreaskarzinom signifikant verlängert ( $p=0,038)$ [1]. Diese Studie führte zur Zulassung von Erlotinib in der Erstlinientherapie des metastasierten Pankreaskarzinoms. Eine interessante Beobachtung in dieser Studie war, dass Patienten mit einem Hautausschlag des Schweregrades $>1$ in besonderer Weise von der zusätzlichen Erlotinib-Gabe profitierten. Die aktuelle Phase-III-Studie AViTA macht deutlich, dass offenbar nicht nur Patienten mit einem Hautausschlag des Schweregrades $>1$ von der Kombination mit Erlotinib profitieren, sondern auch Patienten mit einem Hautausschlag des Schweregrades 1. Da es bei etwa $2 / 3$ der Studienpatienten $\mathrm{zu}$ einem Hautausschlag kam (bei 393 von 607 randomisierten Patienten), bedeutet das, dass bei etwa $2 / 3$ der mit Gemcitabin plus Erlotinib behandelten Patienten mit einer signifikanten und klinisch hochrelevanten Überlebensverlängerung gerechnet werden darf [2].

\section{Quellen}

1 Moore MJ et al: JCO 2007;25:1960-1966.

2 Van Cutsem E et al: ASCO GI 2009, Abstract 117 und mündl. Vortrag.

Weitere Informationen bei

Roche Pharma AG

Dr. H.-U. Jelitto

Emil-Barell-Straße 1, 79639 Grenzach-Wyhlen Tel. +49 7624 14-3715, Fax -3366

www.roche.de

\section{Chronische Lebererkrankungen: Die Fibrose besser verstehen, besser messen und besser behandeln}

Die Chancen stehen gut, dass schon bald effektiv wirksame Antifibrotika zur Behandlung der Leberfibrose verfügbar sein werden. Vorangetrieben werden müssen allerdings die Bemühungen, den Verlauf der Leberfibrose kontrollierbar zu machen und das am besten mit Testverfahren, die nicht invasiv sind und die sich für eine Routinediagnostik eignen.

Der fibrotische Umbau der Leber markiert den Weg in eine chronische Lebererkrankung, die letztlich nicht selten in einer Leberzirrhose und möglicherweise in einem hepatozellulären Karzinom mündet. So früh wie möglich sollte diesem Weg Einhalt geboten werden, weshalb intensiv nach Wirkstoffen gesucht wird, die eine Hemmung der Progression bewirken und im
Idealfall eine Leberfibrose wieder zur Regression bringen.

Zunächst müssen Verfahren entwickelt werden, mit denen sich die Leberfibrose in ihrem Ausmaß zuverlässig und das nicht invasiv, bestimmen lässt. Als mögliche Option bei der Erfassung der Leberfibrose gelten die UltraschallElastographie per Fibro-Scan sowie die hochauflösende MR-Elastographie. Gesucht wird vor allem aber nach Serummarkern, mit denen sich ohne großen Aufwand und zugleich sensitiv der Verlauf der Leberfibrose überwachen lässt.

Sobald solche Messverfahren etabliert sind, werden sich vergleichsweise rasch gezielt Antifibrotika entwickeln und in ihrer klinischen Wirksamkeit und Sicherheit testen lassen. Die Wissenschaftler hoffen auf antifibrotische Therapieeffekte vor allem bei Substanzen, wie Hemmstoffe proliferativ wirksamer Zytokine und Wachstumsfaktoren, die die Signalwege der Fibrogenese beeinflussen. Antifibrotische Effekte versprechen die Hepatologen sich auch von niedrig dosierten Tyrosinkinase-Inhibitoren, wie sie bereits bei onkologischen Erkrankungen eingesetzt werden, sowie von Angiotensin-Hemmstoffen, wie sie bei kardiovaskulären Erkrankungen Anwendung finden.

\section{Quelle}

Falk Workshop

Translational Research in Chronic Liver Disease 29.-30. Januar 2009 in Heidelberg

Weitere Informationen bei

Publicis Health

Guido Balke

Brückenstr. 21, 50667 Köln

Tel. +49 221 912719-551, Fax -751

guido.balke@publicis-health.de

\title{
Ticker+++ Ticker+++ Ticker+++ Ticker+++ Ticker+++ Ticker+++ Ticker+++
}

Leica Microsystems GmbH. Das neue Lasermikrodissektionssystem Leica LMD7000 bietet als einziges einen flexibel einstellbaren Laser, der sich in Leistung und Präzision an die Probe anpassen lässt. Bislang war es nicht möglich, die beiden zentralen Eigenschaften eines Lasers - große Laserenergie für den Schnitt von dickem Material und hohe Pulsrate für genaues und schnelles Schneiden - in einem System zu kombinieren.

Weitere Informationen bei

Leica Microsystems $\mathrm{GmbH}$

Dr. Kirstin Henze

Tel. +49 6441 29-2550, Fax -2527
B. Braun Melsungen AG. Das kurzfristig resorbierbare Nahtmaterial Safil Quick wird durch das neue Safil Quick ${ }^{+}$ersetzt. Lag die 50\%Reißfestigkeit bisher bei 7 Tagen, so wurde diese nun auf 5 Tage verkürzt. Die Dauer der vollständigen Materialabsorption durch Hydrolyse bleibt unverändert bei 42 Tagen. Veränderungen in der Flechtung und Beschichtung des Nahtmaterials sorgen für hohe Knotensicherheit und Gleitfähigkeit.

Weitere Informationen bei

B. Braun Melsungen AG

Kundenservice

info-opm@bbraun
Shire Deutschland GmbH. Mezavant ${ }^{\circledR} 1200$ mg magensaftresistente Retardtablette steht seit dem 1. Januar 2009 auch als N3 mit 120 Tabletten pro Packung zur Verfügung. Neben einer guten Wirksamkeit in der Akuttherapie hat Mezavant ${ }^{\circledR}$ mit patentierter MMX ${ }^{\circledR}$-Technologie (Multi Matrix System) auch Erfolge in der Langzeit-Remissionserhaltung.

Weitere Informationen bei

Media Concept GmbH (GPRA)

Tanja Menges

tm@mediaoncept.de 
Neuroendokrine Tumoren: Neue Studien belegen Kontrolle des Tumorwachstums mit Sandostatin ${ }^{\circledR}$ LAR $^{\circledR}$ sowie hohe Wirksamkeit des oralen mTOR-Inhibitors RAD001

Neuroendokrine Tumoren (NET) gehörten bislang zu den seltenen Krebserkrankungen. Seit kurzem wurde ein deutlicher Anstieg der Inzidenz festgestellt. Heute geht man von einer Neuerkrankungsrate von 2-5 pro 100000 aus, das entspricht 1600-4000 Fällen in Deutschland pro Jahr. Verbesserte diagnostische Methoden sind eine wesentliche Ursache für den Inzidenzanstieg [1]. Da NET häufig erst in einem fortgeschrittenen Stadium diagnostiziert werden, sind sie dann meist nicht mehr operabel und ihre Therapie gilt als schwierig [2-4]. Das Somatostatin-Analogon Octreotid LAR (Sandostatin ${ }^{\circledR}$ LAR $^{\circledR}$ Monatsdepot) verlängert signifikant die Zeit bis zur Tumorprogression bei Patienten mit gut differenzierten NET des Mitteldarms. Dies zeigt eine Interimsanalyse der in Deutschland durchgeführten PROMID* Studie, deren Ergebnisse kürzlich auf dem Gastrointestinal Cancer Symposium der American Society of Clinical Oncology 2009 in San Francisco, USA, vorgestellt wurden [5]

Mit dem oralen mTOR-Inhibitor RAD001 gelingt es sowohl in der Monotherapie als auch in Kombination mit Octreotid LAR das Tumorwachstum bei den meisten Patienten mit gastroenteropankreatischen neuroendokrinen Tumoren (GEP-NET) zu kontrollieren, wie die RADIANT ${ }^{* *}-1$ Studie und weitere Studien belegen $[6,7]$.

Antiproliferativer Effekt von Octreotid LAR nachgewiesen

6 Monate nach Beginn der Behandlung mit dem Somatostatin-Analogon Octreotid LAR reduzierte sich das Risiko der Krankheitsprogression bei Patienten mit gut differenzierten metas- tasierten NET des Mitteldarms signifikant um $66 \%$ im Vergleich zu Placebo ( $\mathrm{p}=0,000072)$, wie die Interimsanalyse der placebokontrollierten, randomisierten, prospektiven Phase-IIIbStudie PROMID zeigt. Octreotid LAR verlängert das progressionsfreie Überleben signifikant von 6 Monaten unter Placebo auf median 14,3 Monate ( $p=0,000072)$. Somit erreichte diese Studie ihren primären Endpunkt bereits in der Interimsanalyse [5].

\section{mTOR-Inhibition: zielgerichtete Wirksamkeit} bei NET

In dem größten jemals bei neuroendokrinen Tumoren aufgelegten Studienprogramm RADIANT werden Wirksamkeit und Sicherheit des oralen mTOR-Inhibitors RAD001 bei NET evaluiert. Der Wirkstoff hemmt kontinuierlich das mTOR-Protein, einen zentralen Regulator von Zellteilung, Zellproliferation und Angiogenese [7].

RADIANT-1, eine multizentrische, internationale Phase-II-Studie, analysierte die Wirksamkeit und Sicherheit von RAD001 als Monotherapie bzw. in Kombination mit Octreotid LAR bei pankreatischen NET nach Versagen einer Chemotherapie. Die randomisierte, doppelblinde, placebokontrollierte, multizentrische, internationale Phase-III-Studie RADIANT-2 untersucht bei Patienten mit fortgeschrittenen NET und einem Karzinoid-Syndrom die Wirksamkeit und Sicherheit der Kombinationstherapie (RAD001 plus Octreotid LAR). In der placebokontrollierten Phase-III-Studie RADIANT3 werden Wirksamkeit und Sicherheit von RAD001 im Vergleich zu «Best Supportive Care» bei Patienten mit fortgeschrittenen pankreatischen NET ermittelt.

RAD001 war generell gut verträglich. Die häufigsten Nebenwirkungen waren Stomatitis (Monotherapie 43,5\%, Kombinationstherapie 48,9\%), Rash (40\% in beiden Gruppen), Diarrhöe (36,5\% Monotherapie, 28,9\% Kombinationstherapie), Fa- tigue (28,7\% Monotherapie, 33,3\% Kombinationstherapie) sowie Übelkeit (Monotherapie 26,1\%, Kombinationstherapie 33,3\%) [7].

Weitere Informationen zu Novartis Oncology, Sandostatin, Sandostatin LAR sowie RAD001 finden Sie im Internet unter www.novartisoncology.de, www.sandostatin.de sowie www.targetmtor.de. Patienten können sich unter www.leben-mit-net.de und www.leben-mit-akromegalie.de informieren.

* PROMID - Placebo-Controlled, Double-Blind, Prospective Randomized Study of the Effect of Octreotide LAR in the Control of Tumor Growth in Patients with Metastatic Neuroendocrine Midgut Tumors.

** RADIANT - RAD001 In Advanced Neuroendocrine Tumors.

\section{Referenzen}

Yao JC et al.: J Clin Oncol 2008;26:3063-3072. 2 Barakat MT et al.: Endocrine-Related Cancer 2004;11118.

Kouvaraki MA et al.: J Clin Oncol 2004:22:4762-4771.

4 McCollum AD et al.: Am J Clin Oncol 2004;27:485-488.

5 Arnold R et al.: ASCO-GI Cancers Symposium 2009, Abstract \#121 und Poster \#A40.

6 Yao JC et al: J Clin Oncol 2008:26:4311-4318.

7 Yao JC et al.: Ann Oncol 2008;19(8):viii166-viii186, Abstract \#508PD.

Weitere Informationen bei

Novartis Pharma GmbH

Dr. Irene Roth

Leiterin Kommunikation Onkologie

Tel. +49 911273 12643, Fax -11020

irene.roth@novartis.com

\section{Ticker+++ Ticker+++ Ticker+++ Ticker+++ Ticker+++ Ticker+++ Ticker+++}

Gilead Sciences GmbH. Die Zulassung von Viread $^{\circledR}$ (Tenofovirdisoproxil als Fumarat) zur Therapie der chronischen Hepatitis $B$ basiert auf 2 großen Phase-III-Studien, die im Dezember 2008 im renommierten New England Journal of Medicine (NEJM) publiziert wurden. Diese Daten belegen $u$. a., dass sich mit Viread ${ }^{\circledR}$ bei mehr als $90 \%$ der Patienten mit einer chronischen Hepatitis $B$ (HBeAg-negativ) eine dauerhafte virologische Kontrolle erzielen lässt.

Weitere Informationen be

3K Agentur für Kommunikation $\mathrm{GmbH}$

Sabine Theobald

info@3k-komm.de
Pfrimmer Nutricia GmbH. Fortimel Compact ist eine hochkalorische Trinknahrung, die eine deutlich höhere Nährstoffdichte aufweist als vergleichbare Produkte. Die erforderliche Trinkmenge wird dadurch reduziert. Dies kommt gerade älteren $\mathrm{Pa}$ tienten zugute, die generell weniger trinken oder häufiger unter Schluckbeschwerden leiden. Die Versorgung der Patienten mit lebenswichtigen Nährstoffen und Kalorien wird optimiert und die Compliance deutlich verbessert.

Weitere Informationen bei

Birke|Partner Kommunikationsagentur

Meike Hallbauer

meike.hallbauer@birke.de
Essex Pharma GmbH. Bislang erfolgte der Einsatz des TNF $\alpha$-Blockers Infliximab (Remicade ${ }^{\circledR}$ ) häufig erst nach immunsuppressiver Vorbehandlung. Jetzt zeigt die SONIC-Studie, dass nicht immunsuppressiv vorbehandelte Morbus-Crohn-Patienten unter einer Mono- oder Kombinationstherapie mit Infliximab signifikant häufiger eine steroidfreie Remission und eine bessere Abheilung der Mukosa erreichen als unter Azathioprin.

Weitere Informationen bei

Medizin \& Markt GmbH

Karla Hickendorf

Tel. +49 89 383930-34 